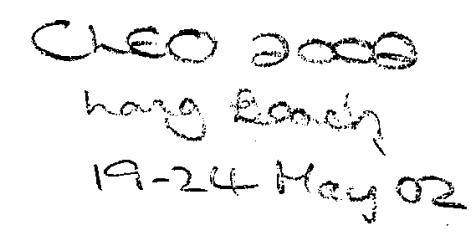

2295

\title{
Power-scaling continuous-wave adaptive laser resonators
}

\author{
David I Hillier, Jason M Hendricks, Stephen J Barrington, David P Shepherd, Robert W. Eason \\ Optoelectronics Research Centre. University of Southampton, Southampton, SO17 IBJ, UK.
}

\author{
Michael J Damzen, Ara Minassian, Benjamin Thompson \\ The Blackett Laboratory, Imperial College, London, SW7 2BZ, UK.
}

We demonstrate a power-scaling strategy in a continuous-wave adaptive laser resonator that actively corrects, via phase-conjugation, for thermally induced phase-distortions introduced by a power-amplifier placed in the output arm of the resonator.

Self-adaptive gain grating lasers have previously been demonstrated that can actively correct for thermally induced phase distortions [1]. Here we discuss the power-scaling of such a resonator which operates at $6 \mathrm{~W}$ in its basic form. Our first attempts using a power amplifier in the output arm have lead to almost $12 \mathrm{~W}$ of single longitudinal mode output with a beam quality of $\mathbf{M}^{2} \sim 1.3$.

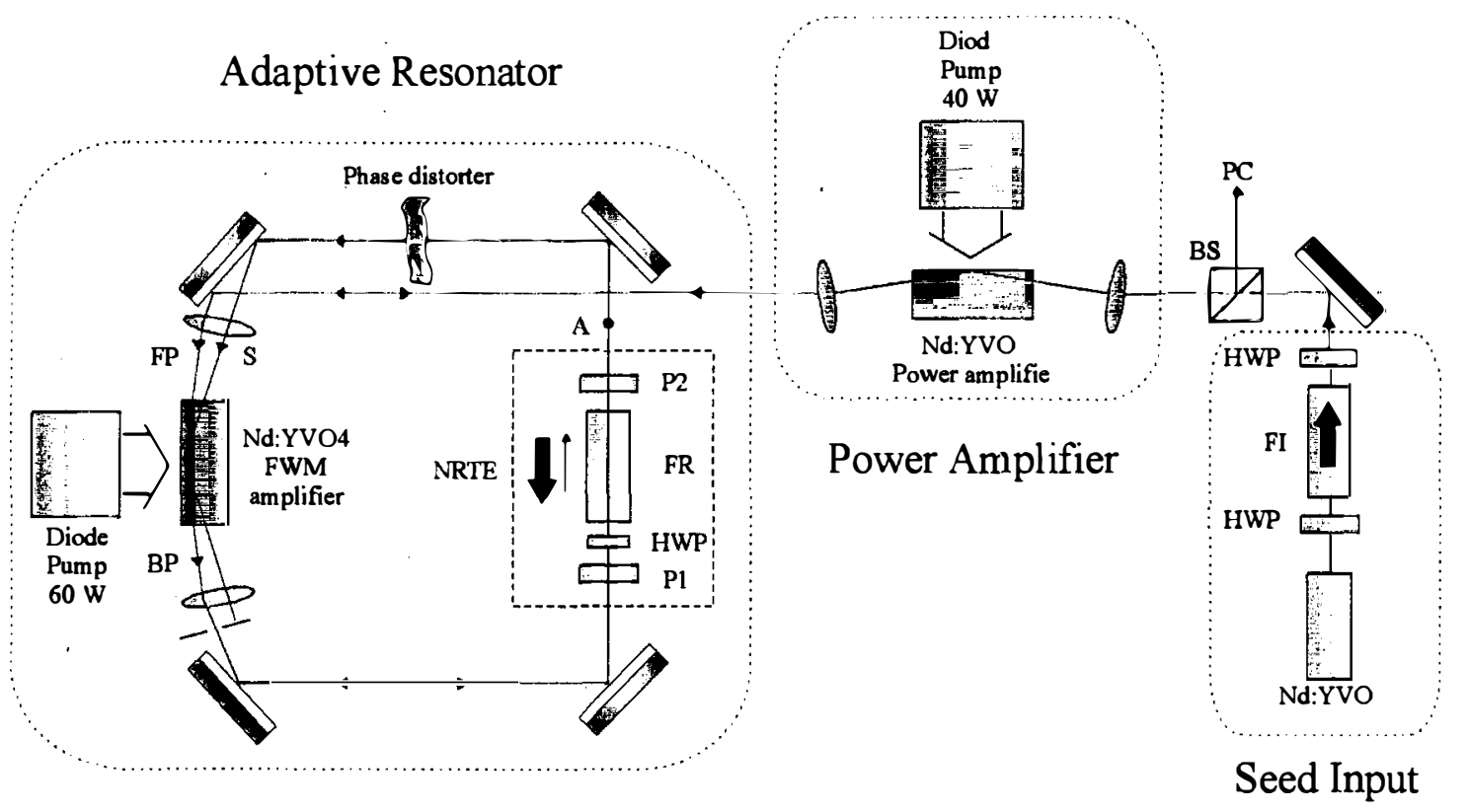

Fig. 1. Schematic of the adaptive laser resonator with a power amplifier in the output anm of the loop. A phasedistorter can be placed in the resonator loop at the position shown whilst leaving the phase-conjugate output unaffected.

Fig. 1. shows a schematic of the adaptive resonator with the power amplifier in the output arm. A seed-laser used to define the operating mode of the system, is launched into the four-wave mixing (FWM) amplifier via the power amplifier. This beam then travels around the resonator so that it intersects with itself back inside the FWM amplifier. The interference pattern produced when the two beams overlap modulates the gain of the FWM amplifier to produce a volume gain-grating [2]. Any phase distortions experienced by the seed laser as it passes around the loop, including distortions from the gain region itself are then encoded within this grating. In the clockwise direction a phase-conjugate lasing mode can build up from amplified-spontaneous emission that is Bragg matched to the volume gain-grating formed. The first-order diffraction from the grating then oscillates in an anti-clockwise 
direction, so that the loop is closed by the grating. The zero order from the grating (the undiffracted part of the phase-conjugate mode) travels back along the original seed beam direction as the phase-conjugate of the seed laser, through the power-amplifier, to become the output, ensuring a high spatial quality beam.

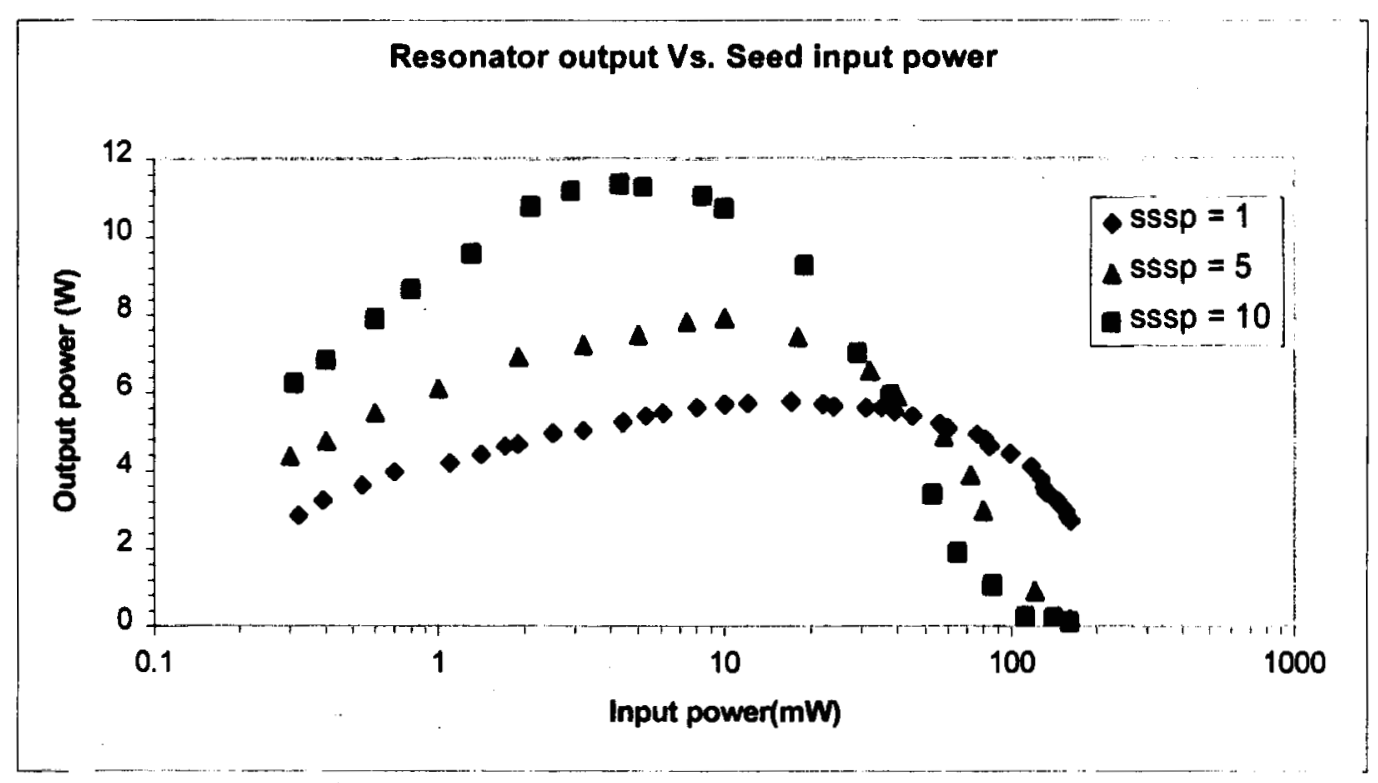

Fig. 2. Graph of the phase-conjugate output power of the combined adaptive resonator and power-amplifier as a function of seed-laser input power. Three plots are shown for SSȘP gains of the power-am plifier of 1, 5 and 10 .

Fig 2. shows the PC output power from the adaptive laser resonator with the power-amplifier in the arm as a function of the seed input power for a range of power amplifier gains. The first curve (diamonds) shows the output when the power-amplifier has a small-signal single-pass (SSSP) gain of 1 . As the input power increases the PC output power also increases. At this stage the contrast ratio of the interference pattern writing the grating in the FWM amplifier is increasing and so saturates the gain giving a well defined grating. The maximum output of $\sim 6 \mathrm{~W}$ from the resonator occurs for an input power of $\sim 20 \mathrm{~mW}$. Beyond this value the PC output begins to fall as the gain is over saturated. The second curve in figure 2. (triangles) show the PC output when the power-amplifier has a SSSP gain of $\sim 5$. The third curve (squares) shows the PC output when the power-amplifier has a SSSP gain of $\sim 10$. The seed input for maximum output occurs at $\sim 4.3 \mathrm{~mW}$ giving a total output power of $11.4 \mathrm{~W}$

[1] M.Trew, G.J.Crofts, M.J.Damzen, J.Hendricks, S.Mailis, D.P.Shepherd, A.C.Tropper, R. W.Eason, "Multiwatt Continuous-wave adaptive laser resonator" Opt. Lett., 25, 1346, (2000).

[2] S.Mailis, J.Hendricks, D.P.Shepherd, A.C.Tropper, N.Moore, R. W.Eason, G.J.Crofts, M.Trew, M.J.Damzen," High-phase-conjugate reflectivity (>800\%) obtained by degenerate four -wave mixing in a continuous-wave diode-side-pumped $\mathrm{Nd}_{\mathrm{YVO}} \mathrm{Y}_{4}$ amplifier" Opt. Lett., 24, 972, (1999). 